Krugman, P. (2010). "When zombies win", "The New York Times, 19. Dezember 2010

Küppers, J-P.; Küppers, E. W. U. (2013). Die Macht weißer Stimmzettel, Zeitschrift für Politikberatung, ZPB, Nomos, Baden Baden.

Küppers, J-P. (2013 a). Gemeinwesenarbeit - eine Tätigkeit an der Gesellschaft. In: Sozial Extra, 37/1-2, S. 40, Springer VS.

Küppers, J.-P. (2013 b). Die Jugend, die wir verloren haben. In: Sozial Extra, 37/11-12, S. 15-19, Springer VS.

Küppers, U. (2013). Denken in Wirkungsnetzen, Tectum, Marburg.

Küppers, U. (2000). Bionik des Organisationsmanagement, iomanagement, 6/2000, 22-31.

Matthäus 25, 29, http://bibel-online.net/buch/luther_1912/matthaeus/25/ Naturschutzbund Deutschland NABU (2013). Eine Welt aus Plastik. Wie Kunststoff unser Leben überschwemmt.

Shell Deutschland Holding (2010). 16. Shell-Jugendstudie 2010, Albert, M. et. al..

Streeck, Wolfgang (2013). Gekaufte Zeit. Die vertagte Krise des demokratischen Kapitalismus. 4. Auflage. Suhrkamp, Berlin.

Thomsen, J. (2013). Naturzerstörung aus der Vogelperspektive, globalmagazin.com, global ${ }^{\circ}$ blog, 4.7.2013, inkl. Landsat-Satteliten-Videos über globaler Veränderungen auf der Erde zw. 1984 und 2012.

Umweltbundesamt UBA (2013). Ein Meer von Kunststoffen, Was können wir gegen die Vermüllung der Ozeane tun?, UBA-Bericht 7.1.2013, s. a. ZDF Doku zum Thema mit UBA-Beteiligung am 8. Juli 13. (www.zdf.d e/ZDFmediathek/beitrag/video/1889616/).

Vogl, Joseph (2010/2011). Das Gespenst des Kapitals. Diaphanes, Zürich Welzer, Harald (2008). Klimakriege - Wofür im 21. Jahrhundert getötet wird. S. Fischer Verlag, Frankfurt am Main.
Wierichs, G. (2012). Das Märchen von der Geldvernichtung, Beitrag auf: www.nachdenkseiten.de, 24.6 .

www.FAZ.net (2013). Italien verstärkt Präsenz im Mittelmeer, 13.10.2013.

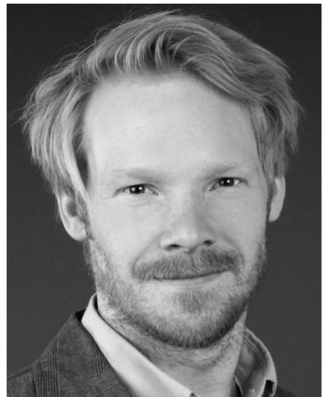

Jan-Philipp Küppers ist Sozialarbeiter, Politikwissenschaftler und Soziologe. Er studierte in Emden, Kiel und Zürich. Sein Interesse gilt Diskursen zu gesellschaftlichen Strukturfragen und eines gerechten Gemeinwesens. Zur Zeit lebt er in Vancouver, Kanada. Email: jan-philipp.kueppers@gmx.de.

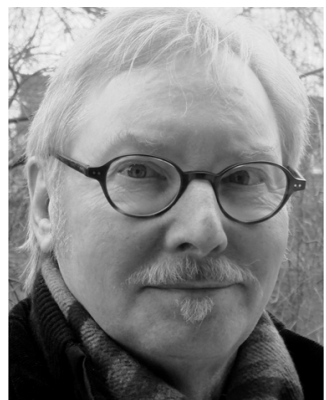

E. W. Udo Küppers ist promovierter Ingenieur, Bioniker und Systemiker. Er studierte Fertigungstechnik, Bionik und Evolutionsstrategie in Düsseldorf und Berlin. Unter KüppersSystemdenken - früher Küppers-Bionik - gilt sein Interesse Arbeiten im Grenzbereich zwischen Natur, Technik und Gesellschaft. Email: mail@udokueppers.de.

\title{
Wirklich gewollt? Wie zu „machen“? Von wem anzugehen?
}

\section{Die Herausforderung des Generationswechsels in den Ortsgruppen von politischen Parteien}

Peter-Georg Albrecht

\begin{abstract}
Kernaussagen
Der Schlüssel zum Gelingen eines Generationswechsels in Ortsgruppen von politischen Parteien liegt wie bei allen Engagiertengruppen in der Anerkenntnis, dass bei einem Generationswechsel zwei Bereiche - der des Nachwuchses und der der Alteingesessenen - zu bedenken sind. Dass ein Generationswechsel für jede politische Engagiertengruppe eine machbare - funktionale - Herausforderung darstellt, aus der sich Leitungsaufgaben ergeben und die - strukturell - sichtbar werden kann, ist der Schlüssel zum Gelingen eines Generationswechsels in Engagiertengruppen. Stets geht es darum, Nachwuchs für sich und die eigene Sache zu gewinnen, gleichzeitig aber auch loslassen zu können.
\end{abstract}

\section{Einleitung}

Vieles deutet darauf hin, dass die Menschen in unserem Land politisch immer aktiver werden. Sie nutzen dazu - unter anderem - das Internet. Sie diskutieren engagiert in Online-Foren über die rechte Art, Politik durchzusetzen, Politik zu vermitteln und Zustimmung zu bekommen und kommen dabei auch auf andere, ihnen je spezifisch wichtige Themen zu sprechen. Sie organisieren Kampagnen. Sie übernehmen Verantwortung für bestimmte Aufgaben. Sie betrachten sich als Freunde. Und sie haben beim Politik machen viel Spaß...

Es gibt Menschen, die sich ganz sicher sind, dass jede neue Generation ihre Aufgaben von der vorherigen Generation gesagt, gelehrt, ja vielleicht sogar eingetrichtert bekommt. Es gibt aber auch Menschen, die der Überzeugung sind, dass jede neue Generation ihre Aufgaben allein, gewissermaßen intuitiv und von innen heraus entdeckt. Und es gibt natürlich auch Menschen, die glauben, jede neue Generation findet zu ihren Aufgaben in der Auseinandersetzung mit der vorherigen Gene- 
ration. Nur für die zuletzt Genannten sind die folgenden Überlegungen niedergeschrieben worden.

Voraussetzung, die folgenden Gedanken zu verstehen, ist allerdings, sich einmal vorzustellen (obwohl genau dies vielleicht manchem fiktiv erscheinen mag), Religion, Kultur, Sport, Wohlfahrtspflege und Politik werde ausschließlich von Engagiertengruppen geschaffen, die diese leben, gestalten und weitergeben; die also Träger der darin enthaltenen Handlungslogiken, Prinzipien und Werte sind; und gäbe keine gesellschaftlichen Rahmen bzw. Institutionen (wie beispielsweise Kirchen, Theater, Sportvereine, Wohlfahrtsverbände oder Parteien), die so etwas wie Religion, Kultur, Gemeinschaftssport, Wohlfahrtspflege und Politik, also alles Dinge, die eigentlich nicht allein praktiziert werden können, nahelegen und so immer wieder zu entsprechenden Gruppengründungen anregen.

Blickt man in die Geschichte des Verbands- und Vereinswesens, verwundert, dass in vielen heutigen Engagiertengruppen (wie die Ortsgruppen von Parteien aus analytischen Gründen im Folgenden genannt werden) die Vorstellung herrscht, ein Generationswechsel könnte darin bestehen, dass die sich lichtenden Reihen der Alteingesessenen durch Neue aufgefüllt werden könnten bzw. zu den altgewohnten Aktivitäten einzelne interessierte Neue hinzustoßen, die sich gewissermaßen hineinfinden, „zwischen die Stühle setzen“ und dann dort wohlfühlen werden. Mit Verlaub: Das war nie so und ist auch in stetig erfolgreich den Generationswechsel vollziehenden Vereinen (Feuerwehren, Fußballvereine) nicht so!

Ein Generationswechsel ist stets eine funktionale Herausforderung einer ganzen parteilichen Engagiertengruppe und insbesondere ihrer Leitung, eine Herausforderung, die entsprechende Strukturen erfordert. Im Folgenden geht es also darum, zu beschreiben, was es braucht, wenn es als wichtig angesehen wird, dass Generationen in einen Austausch und in Auseinandersetzung miteinander stehen und etwas weiterund übergegeben werden soll. So etwas kann in Engagiertengruppen geschehen - allerdings nicht so ideell motiviert wie vielleicht in Familien und auch nicht so einfach mit finanziellen Anreizen zu versehen, wie es vielleicht in Wirtschaftsunternehmen möglich wäre...

Der Text wird denen nicht gerecht, die glauben: Politische Einflussnahme, Politikvermittlung, das Sammeln von Wählerstimmen, aber auch - interessenbedingter - parteiinterner Zusammenhalt, entstehen dann, wenn und weil Individuen spüren, dass es so etwas braucht. Aber er hilft auch denen nur wenig weiter, die der Auffassung sind: Wie Politik zu machen, wie Politik zu vermitteln und der Wähler zu überzeugen wäre und wie dies alles in Bundes-, Landes- und Ortsverbänden mit - je nach föderaler Ebene und regionaler Verortung - sehr unterschiedlichen Themenschwerpunkten zu leben ist, haben ausschließlich die zu bestimmen, die dies zuvor bzw. früher getan haben.

\section{Um was und wen es geht... Generationswechsel zwischen Älteren und Jüngeren}

Der Generationswechsel, von dem wir sprechen wollen, ist vereinfacht gesagt - zunächst ein Wechsel, in eine ältere Generation einer jüngeren Generation etwas weiter- und übergibt. Voraussetzung für einen solchen Wechsel ist die Bereitschaft der Jüngeren zur Übernahme (ein Thema, das hier nicht weiter verhandelt wird), Voraussetzung ist aber auch - und das ist die Kehrseite ein und derselben Medaille - ein Loslassen der Älteren.

Nun ist es - scheinbar - so, dass sich in der alternden Gesellschaft mehr Ältere engagieren. Und es ist so, dass in der alternden Gesellschaft sehr viel mehr Ältere sehr viel weniger Jüngeren etwas weitergeben und übergeben wollen. Stets erscheint die eigene Gruppe in die Jahre gekommen zu sein, während die anderen immer noch ungebunden wirken. $\mathrm{Ob}$ dies nun nur so scheint oder so ist: In den Blick zu nehmen ist, dass die Jüngeren sich heute sehr viel entfernter niederlassen als zu früheren Zeiten, mit höheren „Druck“ bezüglich ihrer Erwerbsarbeit zu kämpfen haben, mehr Zeit als je zuvor im Auto verbringen, in Sachen Partnerschaft und Kindererziehung stärker involviert sind, häufiger Ehrenämter und Hobbies beginnen und auch wieder aufgeben sowie - und das mag vielleicht entscheidend sein - auf mehreren verschiedenen Kanälen (Vis a Vis, per Telefon, per Handy, per SMS, per Email, per Facebook, per Internet) miteinander kommunizieren und Gemeinschaftlichkeit herstellen - zu ihrer Zufriedenheit. Bestehen also gute Voraussetzungen für einen Generationswechsel?

\section{Von was wir sprechen... Ziel, Zweck, Aktivitäten, Strukturen und Vermögen von Engagiertengruppen}

Engagiertengruppen sind keine Familien, aber auch keine Unternehmen, in denen ideelle oder ökonomische Vermögen (geteilte Lebensgeschichten bzw. gut gepflegte Kundenstämme) weiter- und übergeben werden sollen, bezüglich derer es moralisch selbstverständlich oder aber ökonomisch lukrativ für Neue wäre, diese auch zu übernehmen.

Engagiertengruppen bestehen aus einer Mischung von Dienstleistungs- und Mitgliedschaftspraktiken, -logiken und -prinzipien. In Engagiertengruppen finden immer wieder gruppendynamische Aushandlungsprozesse statt, folgen auf entsprechende Konfliktphasen Vertrautheits-, aber auch Individualisierungsphasen. Engagiertengruppen werden - wenn es Menschen notwendig erscheint - gegründet, Engagiertengruppen können aber auch aufgelöst werden (so dass es gar keines Generationswechsels im Sinne der hier vorgestellten Überlegungen bedarf).

Engagiertengruppen bestehen - zumindest zum Zeitpunkt der Gründung - zumeist aus Gleichgesinnten, die ein Ziel bzw. einen Zweck verfolgen, den sie durch ihren Zusammenschluss zu verwirklichen suchen. Immer ergibt sich aus dem Zusammenschluss auch Gemeinschaftlichkeit. Häufig besteht das Vermögen einer Engagiertengruppe aus den sozialen Bezie- 
hungen in ihrem Inneren wie auch in ihrem Außenbezug; ein Vermögen, das nur schwer zu übertragen ist, das sich nur schwer zu eigen zu machen ist und das sich noch viel schwerer genauso oder gar besser wie ein Vorgänger „bewirtschaften“ lässt (allein weil es nie unabhängig der konkreten handelnden Personen und ihren je eigenen Beziehungen zu betrachten ist).

Allerdings muss das Ziel und der Zweck einer Engagiertengruppe und die daraus folgenden Aktivitäten unter dem Gesichtspunkt der Suche nach Vermögenswerten ebenfalls in den Blick genommen werden: Das weiterzugebende Vermögen einer Engagiertengruppe besteht zumeist in Aktivitäten (in deren Ausführung erst - zunächst nicht einschätzbare Gewinne zu ziehen sind), also in Beziehungsarbeit, deren Innenseite (die Beziehungen im Inneren der Gruppe) zumeist voll mit übernommen werden sollen - und deshalb von den Neuen meist - wohl zu Recht - zunächst erst einmal misstrauisch als Hypothek betrachtet werden (ehe anderes erfahrbar ist).

\section{Wie aus Neuen Engagierte und aus Alteingesessenen Ehemalige werden... Funktionale Herausforderungen für einen Generationswechsel}

Soll ein Generationswechsel gelingen, ist - wie oben bereits angerissen - an der Bereitschaft der Jüngeren zur Übernahme von Engagiertengruppenzweck und Engagiertengruppenstruktur zu arbeiten, eine Aufgabe, die das Wort Nachwuchsgewinnung gut umschreibt. Dies erfordert vier - ineinandergreifende und aufeinander aufbauende - Tätigkeiten, die in vielen Engagiertengruppen nicht festgeschrieben sind, nur z.T. sporadisch und informell erbracht werden und über die manche sich schwertun zu sprechen: 1. Die Neuenansprache, 2. die Neuenbegleitung, 3. die Neuengruppenleitung, 4. die Neuengruppenvermittlung.

Soll ein Generationswechsel gelingen, ist ebenfalls an der Bereitschaft der Älteren zum Loslassen zu arbeiten. Dies erfordert (gewissermaßen) die gleichen Tätigkeiten im Inneren der Engagiertengruppe, über die noch viel schwerer zu reden ist als über die der Nachwuchsförderung: die Ehemaligenbetreuung. Darunter zu verstehen ist: 1.) Die Alteingesessenenansprache, 2.) die Alteingesessenenbegleitung, 3.) die Alteingesessenengruppenleitung und 4.) die Alteingesessenenvermittlung.

Neuenansprache bedeutet, Unbekannte, Neugierige und Interessierte, die sich bei bestimmten Anlässen (wie Informationsveranstaltungen, wie Tagen der Offenen Tür) in die Nähe bzw. zwischen die Alteingesessenen „verirrt“ haben, auf gleicher Augenhöhe anzusprechen, sich mit ihnen bekannt zu machen und sie einzuladen. Neuenbegleitung heißt, stetig und wiederholt auf diese Personen zuzugehen und die zunächst flüchtige Bekanntschaft - ähnlich einer Patenschaft - zu vertiefen. Neuengruppenleitung, eine andere Kompetenzen erfordernde und mehr Zeitressourcen erfordernde Tätigkeit, meint, dass Neue zu Gleichaltrigen- bzw. Interessiertenuntergruppen zusammenzubringen sind, die regelmäßig zusammenkommen und beginnen, sich - gut angeleitet - in Zweck und Gemein- schaftlichkeit der Engagiertengruppe hineinzufinden. Neuengruppenvermittlung ist die - konfliktreiche - Tätigkeit, solcherart zusammengefundene Neuenuntergruppen mit den Engagierten und den Alteingesessenen der Engagiertengruppe ins Gespräch und in die Zusammenarbeit zu bringen.

Alteingesessenenansprache heißt, das schwierige Thema Engagementbefristung und -beendigung, die Möglichkeiten und Schwierigkeiten des Loslassens und eines guten Abschlusses und die Perspektiven mit Personen, für die dies (in ihrem wie auch im Interesse der Zukunft der Engagiertengruppe) gut wäre, persönlich zu besprechen. Alteingesessenenbegleitung erfordert, diese Ansprache zu wiederholen (dabei aber nur die Beendigung eines Engagements, keinesfalls die Aufkündigung der gewachsenen Beziehungen im Sinn zu haben). Alteingesessenengruppenleitung ist die Tätigkeit, die Alteingesessenen zu einer Gruppe zusammenzubringen, in der sie - gut begleitet - lernen, Ehemalige zu werden und mit dem Status von ehemaligen Engagierten umzugehen und zu leben. Alteingesessenenvermittlung bedeutet, die Bedürfnisse und Interessen der Alteingesessenen und nun Ehemaligen in die Gesamtgruppe hinein zu vermitteln, so dass - nicht ohne Reibung - Begegnung und hin und wieder auch Kooperation zwischen ihnen und den Engagierten sowie auch den Neuen möglich ist.

\section{Wie eine generative Engagiertengruppe beschaffen sein müsste... Strukturelles Ergebnis eines Generationswechsels}

Werden in einer Engagiertengruppe solcherart Aufgaben (soziologisch: Funktionen) definiert und mit Leben gefüllt, so bildet die Engagiertengruppe (fast wie von selbst) in ihrem Inneren eine Drei-Ebenen-Struktur aus: Die Nachwuchsförderung und Ehemaligenbetreuung ist der Ebene der Engagierten und der Leitung angesiedelt. Auf der Ebene der Neuen findet sich der Nachwuchs, der, zumeist zunächst intuitiv handelnd und an Neuschöpfungen interessiert, sich zu den Engagierten und der Leitung gern zunächst in ein oppositionelles, häufig sogar auch - teils bewusst und strategisch, teils intuitiv, teils sich durch diese Rolle ergebend - provokantes Verhältnis setzt - und dies insbesondere im Blick auf die Alteingesessenen tut. Auf der Ebene der Alteingesessenen finden sich die Ehemaligen, die, aufgrund ihrer Erfahrung, gern ein Kontinuitäten betonendes, häufig forderndes Verhältnis zur Ebene der Engagierten und der Leitung sowie ein distanziertes, häufig abwertendes Verhältnis zur Ebene der Neuen einnehmen.

Mit diesen unterschiedlichen Beziehungs- und Interaktionsmustern umzugehen, erfordert von den Engagierten und der Leitung, die sich für die Neuen wie auch die Alteingesessenen verantwortlich fühlen, ein hohes Maß an Frustrationstoleranz. Sie haben zu ertragen, dass Neue „ihr Ding“ machen und entwickeln und vielleicht auch Nichts vom Bisherigen übernehmen (und dieses so sterben lassen) wollen und Alteingesessene „ihr Ding“ weitertreiben, Gewohntes sichern und häufig Nichts vom Bisherigen übergeben bzw. loslassen wollen. Beide Untergruppen nutzen als Druckmittel den Komplettausstieg. Ankommen, Hineinfinden und Übernehmen ist 
allerdings ebenso schwer, wie auch Gastgeber sein, sich zurücknehmen und loslassen können. Tief im Inneren damit verbundene Ängste auf der einen und Trauer auf der anderen Seite sind zu erkennen, anzunehmen, zuzulassen und zu bearbeiten.

Für die Engagierten ergeben sich aus dem Gesagten Leitungsaufgaben (in einem Verein Vorstandsaufgaben genannt). Neben die Aufgaben der Gruppenleitung, der Einzelmitgliederbetreuung, der Außenvertretung, der Kassenführung und der Vorstandsleitung müssen, soll ein Generationswechsel gelingen, zwei neue Leitungsaufgaben ernst genommen werden: Die der Nachwuchsgewinnung und die der Ehemaligenbetreuung.

Konkret bedeutet das für die Engagierten bzw. die Leitung: Zusätzliche Talente sind zu finden und einzubinden; oder aber als Fähigkeit bei den vorhandenen Leitungspersonen zu entwickeln. Und diese Talente und Fähigkeiten müssen zur Wirkung kommen können und deutlich sichtbar werden. Das bedeutet, sie nicht nur mit Leben zu erfüllen, sondern auch im Aufgabenspektrum wie auch im Strukturplan der Engagiertengruppe zu verankern. Mit anderen Worten: Es dürfte keine Leitungssitzung mehr geben, bei der der Nachwuchsgewinnung wie auch der Ehemaligenbetreuung nicht eben so viel Zeit gewidmet wird wie bspw. dem Vorstandsvorsitz, den Finanzen, den Kooperationspartnern, der Betreuung einzelner Mitglieder und der Leitung der gesamten Engagiertengruppe mit ihren spezifischen Zielen.

\section{Zusammengefasst und in Merksätzen formuliert...}

Vielleicht noch einmal mit anderen Worten und insbesondere für die Alteingesessenen und Engagierten gesagt, was zu beachten ist, widmet sich eine Engagiertengruppe wirklich dem Generationswechsel.

6.1 Alle sind gefragt. Zunächst kommt es darauf an, dass sich eine Engagiertengruppe darauf verständigt, den Generationswechsel anzupacken.

6.2 Veränderung ist jetzt wichtiger als Festigung. Es mag einfach klingen, aber: Eine Engagiertengruppe muss sich damit anfreunden, dass mit der Arbeit an einem Generationswechsel eine Zeit der Veränderung anbricht. Auf Altbekanntes zurückzugreifen, Altbewährtes zu tun und dies im Namen von Alteingesessenen und mit dem Ziel der Festigung, ist nun nicht mehr möglich.

6.3 Es gilt zu streiten; ruhig geht es nun nicht mehr zu. Der Generationswechsel verunsichert insbesondere die Alteingesessenen. Die Engagierten und die Leitung, die einen Generationswechsel vorantreibt, muss sich auf Gegenwind einstellen und für diese Sache kämpfen. "Ruhig machen" lässt sich so etwas Existenzielles nicht.

6.4 Vieles ist zu bedenken; es kann nicht nur in eine Ecke geschaut werden. Ein Generationswechsel fordert dazu heraus, Ziel, Zweck, Aktivitäten, Strukturen und Vermögen von Engagiertengruppen in den Blick zu nehmen. $\mathrm{Zu}$ fragen ist: Was sollte auf jeden Fall erhalten werden, was kann gelassen werden?
6.5 Der Nachwuchs ist zu gewinnen, Alteingesessene sind zu begleiten. Es gilt zu klären, wer überhaupt eine gewinnende Art hat. Es ist zu überlegen, wer Alteingesessenen gut dabei unterstützen kann, zu Ehemaligen zu werden, ohne dass sie in die Isolation "entlassen" werden.

6.6 Leitung wird nur durch Leiten erlernt. Haben die Engagierten über die Nachwuchsgewinnung und die Ehemaligenbetreuung hinaus im Sinne, auch die Leitung der Engagiertengruppe zu erneuern, müssen sie die dafür in Frage kommenden Personen immer wieder aktiv "vorn" hinstellen. Das erfordert eine klare Ansprache derselben. Das erfordert, sich zurückzunehmen. Das erfordert aber auch, den nun vorn Stehenden die Zustimmung der Engagiertengruppe zu beschaffen.

6.7 Leiter bedürfen klarer Leitungsaufträge, aber auch des Rückhalts der "Grauen Eminenzen". Neue Leiter, die beim Leiten auf Probe Zuspruch erfahren, lassen sich nach einer gewissen Zeit verantwortlich mit der Leitungsaufgabe beauftragen. Die vormaligen Leiter müssen nun in die zweite Reihe wechseln. Da sie jedoch noch eine ganze Zeit lang von vielen informell als "die eigentlichen" Führungskräfte angesehen werden, haben sie ihre Zeit darauf zu verwenden, lautstark, ehrlich, verlässlich und kontinuierlich für die neue Leitung, ihren Stil und ihre Inhalte zu werben.

6.8 Ganz allein lässt sich ein Generationswechsel wohl nicht stemmen. Engagiertengruppen, die sich diesem schwierig zur Sprache zu bringenden und vor allem mit ihrem Innenleben abhängenden Thema befassen, dürfen sich nicht scheuen, externe Begleitung in Anspruch zu nehmen. Denn nur unabhängige Berater sind in der Lage, Engagiertengruppen den Spiegel vorzuhalten sowie die Wege des Generationswechsel aufzuzeigen, von denen ja im Grunde alle wissen, wie sie zu gehen sind (weil sie aber auch wissen, dass es schwer und schmerzlich ist, sie zu gehen).

\section{Eine solche Veränderung lohnt sich, denn...}

Ein Generationswechsel, der die Alten nicht im Blick hat und ihre Entlassung und Desintegration in den Kauf nimmt, ist ebenso unsolidarisch wie ein Generationswechsel, bei dem die Jungen vereinnahmt werden sollen.

Die Anerkenntnis, dass bei einem Generationswechsel zwei Bereiche, die des Nachwuchses und die von Ehemaligen zu bedenken sind, dass ein Generationswechsel für jede politische Engagiertengruppe eine machbare - funktionale - Herausforderung darstellt, aus der sich Leitungsaufgaben ergeben und die - strukturell - sichtbar werden kann, ist der Schlüssel zum Gelingen eines Generationswechsels in Engagiertengruppen.

Scheitern kann er allerdings auch; ist es doch manchmal besser, mit etwas aufzuhören, etwas aufzugeben, vielleicht, weil es andere längst - anders, passender oder vielleicht sogar besser - tun.

Es kann ja sein, dass nur wir glauben, dass Politikdurchsetzung, Politikvermittlung, Zustimmungsgewinnung sowie die damit verbundene parteigenossenschaftliche Gemeinschaftlichkeit in Bundes-, Landes- und Ortsverbänden von 
Parteien seinen Platz hat. Vielleicht meinen nur wir, entscheidend sei die Vis-a-Vis-Kommunikation beim Politik machen und beim Austausch über Politik. Es kann natürlich sein, dass wir erfahren haben, wie gut das tut. Aber kann es nicht auch sein, dass wir uns einbilden, dass nur wir wissen, wie Politik richtig zu machen ist - und wie man sich dabei wohlfühlt?

\section{Nachtrag}

Generationen unterscheiden sich. Das kann einen Generationswechsel erschweren.

Jüngere bewerten eine Aktivität, die sie übernehmen sollen, aus der Situation heraus, sie setzen sie in ein Verhältnis zu ihren Fähigkeiten und Begrenzungen und überlegen, wie andere - gleichaltrige - Personen wohl entscheiden und was sie zur Übernahme dieser Aktivität sagen würden; Lebenserfahrung spielt dabei nur eine geringe Rolle.

Sie wissen, das mit der Aktivität etwas auf sie zukommt. Deshalb prüfen sie auch das Verhältnis der Aktivität zu den anderen Aktivitäten und Bereichen ihres Lebens, dem so u.a. dem Wohnen, der Mobilität, der Erwerbsarbeit, der Partnerschaft und Familie, der Ehrenämter und Hobbies, den Freundschaften, der Kommunikation. Als sicher kann gelten: Sie wohnen unabhängig, fahren viel und gern, halten den Stress auf der Arbeit für normal, widmen dem Lebensgefährten und den Kindern, anderen Ehrenamtlichen und Hobbie-Partnern sowie ihre Freunden viel Zeit und kommunizieren mit ihnen, wo auch immer sie sich aufhalten; auf vielerlei Art und Weise.

Ältere bewerten eine Aktivität, die sie übergeben wollen, eher anhand der Gewinne, die sie ihrer Erinnerung nach aus der Ausführung der Aufgabe gezogen haben. Weil sie sie überund aufgeben wollen, entfallen auf ihrer Seite Überlegungen zu Passungen zu Lebenssituation, Fähigkeiten und Begrenzungen. (Allenfalls wird überlegt, was die Anderen dazu sagen, dass man aufhört.)

Die Älteren haben eine Entlastung im Sinn. Sie schätzen die Wirkung der Aufgabenaufgabe auf ihre sonstigen Lebensbereiche und -aufgaben, so z.B. das Wohnen, die Mobilität, die Partnerschaft und die Beziehungen zu den Kindern, die die Ehrenämter und Hobbies, die Freundschaftsbeziehungen; einzig die Erwerbsarbeit spielt im Alter keine bzw. nur eine geringe Rolle. Als sicher kann gelten: Sie wohnen gern nah bei den ihren, halten Fahrten für eine Notwendigkeit, um zu einem Ziel zu kommen, (lassen sich eben nicht mehr von Erwerbsarbeit stressen), verbringen mit dem Lebensgefährten viel, mit den Kindern weniger Zeit, haben - überschaubar und geregelt, ja häufig ritualisierte - Kontakte zu Ehrenamtlichen, Hobbie-Partnern, aber auch Freunden - vorzugsweise Vis a Vis bzw. per Telefon, nur hin und wieder auch schon per Email.

Generationen unterscheiden sich... Aber ist möglicherweise allein die Differenz zwischen Wollen und das Sollen das größte Problem?

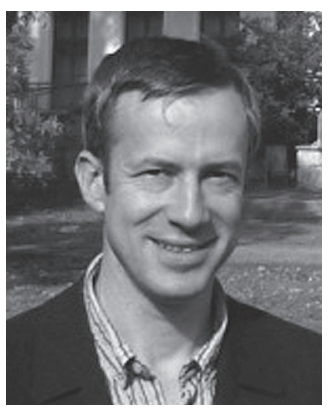

Dr. Peter-Georg Albrecht ist Sozialund Alterswissenschaftler an der Hochschule Magdeburg-Stendal im Fachbereich Sozial- und Gesundheitswesen

Email: peter-georg.albrecht@hsmagdeburg.de

\section{Die politischen Parteien der Bundesrepublik Deutschland}

Geschichte - interne Entscheidungsprozesse - strategische Potentiale Herausgegeben von Karl-Rudolf Korte, (NRW School of Governance, Universität Duisburg-Essen)

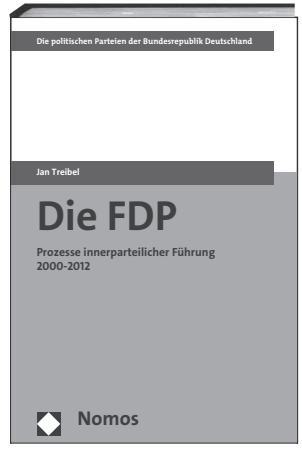

Die FDP

Prozesse innerparteilicher Führung 2000-2012

Von Jan Treibel

2014, 283 S., brosch., 22,90€ ISBN 978-3-8329-5386-7 www.nomos-shop.de/12279

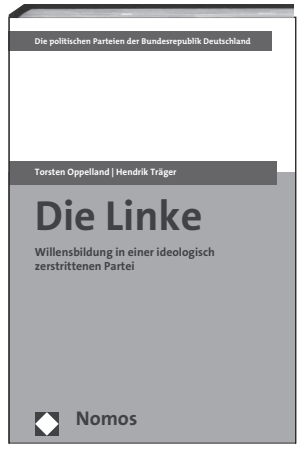

Die Linke

Willensbildung in einer ideologisch zerstrittenen Partei Von Torsten Oppelland und Hendrik Träger 2014, 263 S., brosch., 19,90€ ISBN 978-3-8329-6965-3 www.nomos-shop.de/14064 